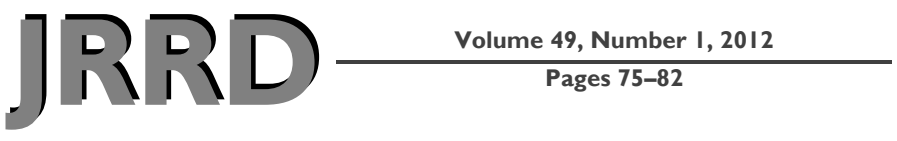

\title{
Pilot study for quantifying driving characteristics during power wheelchair soccer
}

\author{
Amit Kumar, MS, PT; ${ }^{1-2}$ Amol M. Karmarkar, PhD; ${ }^{3}$ Diane M. Collins, PhD; ${ }^{2}$ Ana Souza, MS, PT; ${ }^{1-2}$ Michelle L. \\ Oyster, MS; ${ }^{1,4}$ Rosemarie Cooper, MPT; ${ }^{1-2}$ Rory A. Cooper, PhD ${ }^{1-2,4^{*}}$ \\ ${ }^{1}$ Human Engineering Research Laboratories, Department of Veterans Affairs, Pittsburgh, PA; ${ }^{2}$ Department of Rehabilita- \\ tion Science and Technology, University of Pittsburgh, Pittsburgh, PA; ${ }^{3}$ Division of Rehabilitation Sciences, University of \\ Texas Medical Branch, Galveston, TX; ${ }^{4}$ Department of Physical Medicine and Rehabilitation, University of Pittsburgh, \\ Pittsburgh, $P A$
}

\begin{abstract}
This study determined the driving characteristics of wheelchair users during power wheelchair soccer games. Data for this study were collected at the 28th and 29th National Veterans Wheelchair Games. Nineteen veterans who were 18 years or older and power wheelchair soccer players completed a brief demographic survey and provided information about their power wheelchairs. A customized data-logging device was placed on each participant's wheelchair before power soccer game participation. The data logger was removed at the end of the final game for each participant. The average distance traveled during the games was $899.5+/-592.5 \mathrm{~m}$, and the average maximum continuous distance traveled was $256.0+/-209.4 \mathrm{~m}$. The average wheelchair speed was $0.8+/-0.2 \mathrm{~m} / \mathrm{s}$, and the average duration of driving time was $17.6+/-8.3 \mathrm{~min}$. Average proportion of time spent at a speed $>1 \mathrm{~m} / \mathrm{s}$ was $30.7 \%+/-33.8 \%$, between 0.5 and $1 \mathrm{~m} / \mathrm{s}$ was $16.2 \%+/-34.4 \%$, and $<0.5 \mathrm{~m} / \mathrm{s}$ was $21.4 \%+/-$ $24.3 \%$. The information from this descriptive study provides insight for future research in the field of adapted sports for people with high levels of impairments who use power wheelchairs for their mobility.
\end{abstract}

Key words: activity, adapted sports, data logger, disability, mobility, National Veterans Wheelchair Games, power wheelchair, rehabilitation, veterans, wheelchair users.

\section{INTRODUCTION}

Participation in adapted sports has contributed significantly to the quality of life of veterans with disabilities
[1-2]. Sports and recreation are of the utmost importance for maintaining or increasing functional capacity and psychological well-being for people with physical disabilities [3-4]. Regular physical activity has been found to reduce the risk of secondary complications and comorbidities associated with injury or disease and to increase functional capacity $[3,5]$. However, individuals with higher levels of physical impairment have difficulty participating in regular physical activity because of various limitations [6]. Therefore, individuals who rely on a power wheelchair (PWC) are at risk for adopting a sedentary lifestyle because of a lack of recreation and limited physical activity [6]. A paucity of literature exists on PWC use during sports and other mobility-related activities.

Power soccer is a team-based sport for people with severe disabilities that is gaining popularity among individuals who rely on a PWC for mobility $[5,7]$. The performance

\footnotetext{
Abbreviations: $\mathrm{CP}=$ cerebral palsy, HERL $=$ Human Engineering Research Laboratories, MWC = manual wheelchair, NVWG = National Veterans Wheelchair Games, PWC = power wheelchair, SCI = spinal cord injury, VA = Department of Veterans Affairs.

*Address all correspondence to Rory A. Cooper, PhD; VAPHS-Human Engineering Research Laboratories, 6425 Penn Ave, Suite 400, Pittsburgh, PA 15206; 412-822-3700; fax: 412-822-3699. Email: rcooper@pitt.edu http://dx.doi.org/10.1682/JRRD.2010.09.0191
} 
and quality of the play depends on a fast response, speed, turning radius, sound braking control, and strategic passing and positioning abilities [7-8]. Power soccer is a contact sport and each player's wheelchair is equipped with a foot guard to protect the player from impact injury. Little information is available on the mobility patterns and demographic characteristics of individuals who play power soccer. This type of information could be vital for customizing PWCs and their associated components, battery designs, and other specifications to enhance athletic performance and reduce risk of injury during PWC sports [9-10].

In the past, the majority of research was conducted on wheelchair use during sport or activity with nondisabled participants. For example, Reid and Prupas conducted a literature review of 436 articles from 1986 to 1996 related to disability sports [11]. After analyzing the literature, they found that the majority of research was conducted with nondisabled athletes and the results were then compared with individuals with disabilities [11]. Participation in adapted sports has been rising steadily as a result of new innovations and the growth of science and technology, with better assistive devices to enhance functional capacity in individuals with disabilities [2,12]. Advancements in science and technology have produced adapted equipment that allows individuals with disabilities to achieve or even surpass records held by nondisabled athletes [2,12-13]. The role of technology in measuring performance in adapted sports is growing, which could enhance the training efficiency.

Limited research has been conducted on the driving characteristics of PWC users in adapted sports. Several studies have been conducted on the driving characteristics of PWC users during community activities. Sonenblum et al. [14], in a 2008 study of persons who used PWCs in their homes and communities, found that the participants traveled a median of $1.085 \mathrm{~km}$ over $58 \mathrm{~min}$. Daily distance traveled and time spent wheeling varied widely between and across study participants. The measure of wheelchair occupancy presented with the least variation [14]. Cooper et al. found that veterans participating in the National Veterans Wheelchair Games (NVWG) traveled longer distances at higher speeds than civilians traveling in their communities [9]. In addition, this study found that participants traveled at a speed considerably less than the wheelchair's maximum speed (approximately $2.7 \mathrm{~m} / \mathrm{s}$ ) most of the time. In another pilot study, Cooper et al. used customized data loggers to quantify the mobility patterns of children using manual wheelchairs (MWCs) and PWCs in community settings [15]. Data analyzed from the customized data-logging devices revealed that the children who used PWCs drove $1,752.42 \mathrm{~m} / \mathrm{d}$ at a speed of $0.75 \mathrm{~m} / \mathrm{s}$. Ferretti also used wheelchair data loggers to measure the mobility patterns of people using either MWCs or PWCs [16]. Significant correlations between average driving minutes and community participation were found among people using PWCs. A study by Sporner et al. quantified the activity of MWC users during wheelchair basketball and rugby [17]. During the sports activities, wheelchair rugby athletes were found to travel approximately $2,300 \mathrm{~m}$ at $1.33 \mathrm{~m} / \mathrm{s}$ and the wheelchair basketball players traveled approximately 2,700 $\mathrm{m}$ at $1.48 \mathrm{~m} / \mathrm{s}$ [17].

The U.S. Paralympics Committee has given high priority to research on disability sports [18]. An evidencebased approach is needed to determine factors that may affect the performance of power soccer players. Data derived from this approach may improve the prescription criteria and ensure that appropriate PWC designs are being issued to the individuals with disabilities engaged in organized competitive sports. To support the evidencebased approach, the purpose of this pilot investigation was to characterize wheelchair-related mobility patterns of athletes during power soccer games.

\section{METHODS}

\section{Participants}

The Human Engineering Research Laboratories (HERL), a joint research venture between the Department of Veterans Affairs (VA) Pittsburgh Healthcare System and the University of Pittsburgh, has permission to collect research data during the NVWG. Centrally located booths were set up for subject recruitment. Veterans who approached the booths were told about the research being conducted at the NVWG and were invited to participate.

The data for this study were collected at the 28th NVWG held in Omaha, Nebraska, in 2008 and the 29th NVWG held in Spokane, Washington, in 2009. To be eligible, participants had to be 18 years or older and participate in power wheelchair soccer at the NVWG.

\section{Instrumentation}

A custom data-logging device (Figures 1 and 2) was used to monitor PWC usage during soccer games. These caster data loggers were designed and developed at HERL and have been used in multiple studies to assess movement (Figure 2). The front or back casters of participants' PWCs were replaced with a caster wheel with a data logger in its 


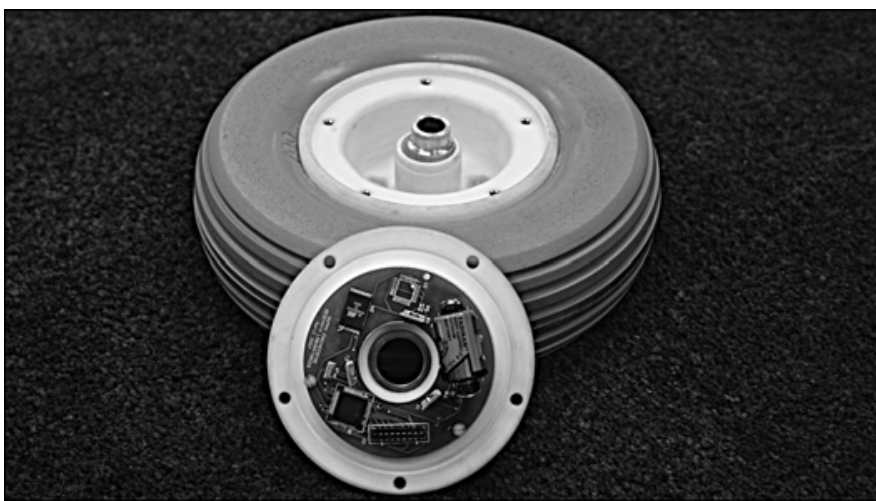

Figure 1.

Custom data-logging device used to monitor power wheelchair usage during soccer games.

hub. The device measures wheel rotations through use of three reed switches mounted $120^{\circ}$ apart on a circuit board and magnets mounted on the side of a custom-designed axle insert. Every time the wheelchair wheel rotated more than $120^{\circ}$, the magnet triggered one of the reed switches. As each reed switch was triggered, a date and time stamp of that event was recorded in the device.

As a participant exited the tournament, the caster containing the data logger was removed and the participant's original caster was put back on his or her wheelchair. Time stamp data from the data-logging devices were used to calculate the following variables: (1) average distance traveled, (2) average speed, (3) maximum continuous distance traveled, (4) duration of driving, and (5) proportion of time spent at different speed levels. For this study, only the first two recorded games were used, because each participant played a different number of games. However, not all participants were able to stay in the tournament to record two or more games worth of data.

Terms used in this analysis are defined as follows: "duration of driving" is the total amount of time the wheelchair was in motion, "stop" is a pause in motion lasting $2 \mathrm{~s}$ or more, "average speed" is the figure represented by the duration of driving divided by the total distance driven, "maximum continuous distance" is the maximum distance traveled between two stops, and "maximum continuous speed" is the maximum speed traveled between two stops.

\section{Study Protocol}

Demographic information was collected on each participant by means of a survey that asked the participant's

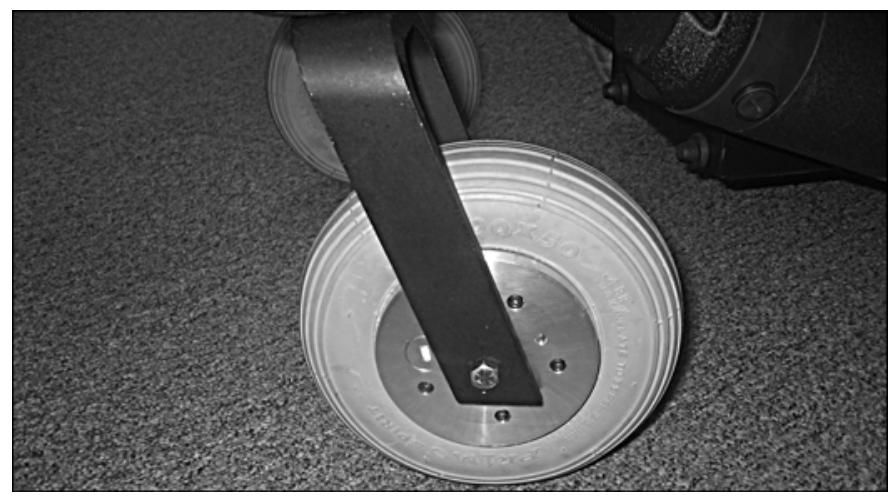

Figure 2.

Custom caster data-logger designed to assess power wheelchair movement.

age, disability, years since injury or diagnosis, PWC type, training habits, and participation in other organized sports. A customized data-logging device was placed on each participant's PWC before his or her respective power soccer games. The start and end times of the power soccer games were recorded and used to calculate the mobility patterns for each game.

\section{Data Analysis}

For the analysis of the demographic data, categorical variables such as disability, sex, and race/ethnicity were represented with frequencies and percentages and continuous variables such as age and years since diagnosis or injury were represented with means and standard deviations. Activity levels during power soccer games could not be calculated on the basis of different users' interfaces, since only one participant used a chin-control joystick. Based on the results of previous studies that characterized the mobility patterns of wheelchair users in the community and during sports, the proportion of time spent by participants at three different speed levels was classified into high, medium, and low levels. Speeds more than $1 \mathrm{~m} / \mathrm{s}$ were classified as high, speeds between 0.5 and $1 \mathrm{~m} / \mathrm{s}$ were classified as medium, and speeds less than $0.5 \mathrm{~m} / \mathrm{s}$ were classified as low. The analyses were done with SPSS version 15 (SPSS Inc; Chicago, Illinois).

\section{RESULTS}

The average (mean \pm standard deviation) participant age was $56.8 \pm 9.1 \mathrm{yr}$, and the average time since injury or the onset of disability was $26.6 \pm 13.8$ yr. Of the 
19 participants, 18 (94.7\%) were male. Thirteen (68.4\%) had a spinal cord injury (SCI), three (16.0\%) had multiple sclerosis, and three (16.0\%) had had a stroke. Sixteen participants (84.0\%) used rear-wheel drive PWC, and three (16.0\%) used mid-wheel drive PWC. Demographic characteristics are presented in detail in Table $\mathbf{1 .}$

The total duration of a power soccer game is $40 \mathrm{~min}$, consisting of two 20 min periods. Table 2 provides details of each participant's mobility patterns during the power soccer games. The overall average distance traveled by the 19 athletes was $899.5 \pm 592.5 \mathrm{~m}$, with a maximum distance traveled of 2,055.87 $\mathrm{m}$. The average speed of all participants during games was $0.8 \pm 0.2 \mathrm{~m} / \mathrm{s}$. The average maximum continuous distance traveled was $256.0 \pm 209.4 \mathrm{~m}$. The average driving time of participants during games was $17.6 \pm 8.3 \mathrm{~min}$. Figure 3 shows the proportion of time each

Table 1.

Summary of demographic characteristics for 19 power wheelchair soccer players at 2008 and 2009 National Veterans Wheelchair Games.

\begin{tabular}{|c|c|}
\hline Variable & Value \\
\hline$\overline{\text { Age, Mean } \pm \text { SD }}$ & $56.8 \pm 9.1$ \\
\hline $\begin{array}{l}\text { Years Postinjury or Onset of } \\
\text { Disability, Mean } \pm \text { SD }\end{array}$ & $26.6 \pm 13.8$ \\
\hline \multicolumn{2}{|l|}{ Disability, $n$ (\%) } \\
\hline $\operatorname{SCI}\left(C_{3-4=6}, C_{5-6=7}\right)$ & $13(68.4)$ \\
\hline Multiple Sclerosis & $3(15.8)$ \\
\hline Other & $3(15.8)$ \\
\hline \multicolumn{2}{|l|}{ Race/Ethnicity, $n$} \\
\hline White & 15 \\
\hline Nonwhite & 4 \\
\hline \multicolumn{2}{|l|}{ Sex, $n(\%)$} \\
\hline Male & 18 (94.7) \\
\hline Female & $1(5.3)$ \\
\hline \multicolumn{2}{|l|}{ Wheel Drive, $n$ (\%) } \\
\hline Rear-Wheel Drive & $16(84.0)$ \\
\hline Mid-Wheel Drive & $3(16.0)$ \\
\hline \multicolumn{2}{|l|}{ Residence, $n$} \\
\hline Rural & 4 \\
\hline Urban & 5 \\
\hline Suburban & 10 \\
\hline \multicolumn{2}{|l|}{ Type of Joystick, $n$} \\
\hline Hand Control & 18 \\
\hline Chin Control & 1 \\
\hline Training Time, h & 4.6 \\
\hline
\end{tabular}

participant spent at the three different speed levels. The average proportion of time spent at the high speed $(>1 \mathrm{~m} / \mathrm{s})$ was $30.4 \pm 33.9$ percent, the average proportion of time spent at the medium speed $(0.5-1 \mathrm{~m} / \mathrm{s})$ was $16.3 \pm 34.4$ percent, and the average proportion of time spent at the low speed $(<0.5 \mathrm{~m} / \mathrm{s})$ was $21.5 \pm 24.3$ percent.

\section{DISCUSSION}

The results of our pilot study quantify the mobility patterns of power soccer athletes using data-logging devices on their wheelchair. The data gathered by these devices allowed us to calculate average distance traveled, speed, maximum continuous distance, and proportion of time traveled at three different speed levels during power soccer games.

To our knowledge, this is the first study to measure the mobility characteristics of PWC during power soccer game participation. Results of this study show that the mobility characteristics varied between power soccer participants. These results are consistent with previous data collected on the driving characteristics of PWC users in the community [14-16]. The current study found that the distance traveled by power soccer players ranged from 69.15 to $2,055.87 \mathrm{~m}$, with a mean of $899.5 \pm 592.5 \mathrm{~m}$. Power soccer players were found to travel at an average speed of $0.8 \pm 0.2 \mathrm{~m} / \mathrm{s}$ for an average driving time of $17.6 \pm 8.3 \mathrm{~min}$ (range 2.52 to $31.27 \mathrm{~min}$ ). The results of the power soccer mobility characteristics are not consistent with the activity measured during wheelchair basketball and rugby [17]. Participants in the wheelchair rugby and basketball games tended to travel farther and faster than the power soccer players. Future studies should investigate the factors that influence mobility characteristics during wheelchair sports.

Barfield et al. monitored heart rate among people with SCI, cerebral palsy (CP), and muscular dystrophy competing in a national power soccer tournament [5]. They determined that disability type did influence heart rate responses during PWC sports, since the median heart rate responses for athletes with $\mathrm{CP}$ was significantly higher $(p<0.01)$ than athletes with SCI. In future research studies, it would be interesting to collect both heart rate and mobility characteristics of athletes using PWCs during sports to investigate whether heart rate is related to mobility patterns. Further, the PWC data logger may be an effective measuring device to monitor training 
Table 2.

Participant characteristics and average mobility patterns during power wheelchair soccer games.

\begin{tabular}{|c|c|c|c|c|c|c|c|c|c|}
\hline ID & $\begin{array}{l}\text { Age } \\
\text { (yr) }\end{array}$ & $\begin{array}{c}\text { Body } \\
\text { Weight } \\
\text { (kg) }\end{array}$ & $\begin{array}{c}\text { Games } \\
\text { Played } \\
\text { (No.) }\end{array}$ & Wheel Drive & Diagnosis & Distance (m) & $\begin{array}{l}\text { Speed } \\
(\mathrm{m} / \mathrm{s})\end{array}$ & $\begin{array}{c}\text { Max } \\
\text { Continuous } \\
\text { Distance (m) }\end{array}$ & $\begin{array}{l}\text { Drive Time } \\
\text { (min) }\end{array}$ \\
\hline 1 & 62 & 225 & 2 & RWD & SCI & $1,053.84$ & 0.60 & 187.36 & 28.56 \\
\hline 2 & 52 & 120 & 2 & MWD & MS & 902.33 & 0.48 & 176.35 & 31.27 \\
\hline 3 & 48 & 180 & 2 & RWD & SCI & 819.24 & 0.74 & 279.71 & 17.74 \\
\hline 4 & 54 & 150 & 1 & RWD & MS & $1,620.39$ & 1.09 & 591.77 & 24.77 \\
\hline 5 & 38 & 195 & 2 & RWD & SCI & 69.15 & 0.45 & 15.32 & 2.52 \\
\hline 6 & 63 & 164 & 2 & RWD & MS & 341.52 & 0.78 & 111.50 & 6.64 \\
\hline 7 & 77 & 150 & 2 & RWD & Other & $2,055.87$ & 1.29 & 464.20 & 26.48 \\
\hline 8 & 61 & 250 & 2 & MWD & SCI & 484.73 & 0.70 & 140.65 & 11.42 \\
\hline 9 & 53 & 165 & 2 & RWD & SCI & 825.62 & 0.96 & 192.04 & 14.37 \\
\hline 10 & 60 & 177 & 2 & RWD & SCI & $1,926.07$ & 1.16 & 516.86 & 27.69 \\
\hline 11 & 51 & 155 & 1 & RWD & SCI & $1,426.54$ & 1.28 & 779.66 & 18.49 \\
\hline 12 & 67 & 135 & 2 & RWD & SCI & $1,629.65$ & 1.22 & 442.17 & 25.01 \\
\hline 13 & 68 & 230 & 2 & RWD & SCI & 519.31 & 0.73 & 126.71 & 11.53 \\
\hline 14 & 53 & 180 & 1 & RWD & Other & 428.34 & 0.51 & 79.37 & 13.83 \\
\hline 15 & 62 & 250 & 1 & MWD & SCI & 333.86 & 0.75 & 176.61 & 7.36 \\
\hline 16 & 53 & 190 & 2 & RWD & SCI & $1,046.71$ & 0.77 & 218.11 & 22.28 \\
\hline 17 & 62 & 240 & 2 & RWD & Other & 401.85 & 0.47 & 55.75 & 14.01 \\
\hline 18 & 50 & 140 & 2 & RWD & SCI & 602.41 & 0.69 & 156.29 & 14.20 \\
\hline 19 & 45 & 239 & 2 & RWD & SCI & 604.32 & 0.59 & 155.12 & 16.73 \\
\hline
\end{tabular}

Note: Total duration of time for each game is 40 minutes.

ID = identification, Max = maximum, MS = multiple sclerosis, MWD = mid-wheel drive, RWD = rear-wheel drive, SCI = spinal cord injury.

and provide a method to investigate whether a correlation exists between data-logger data and game performance.

Bumpers are applied to the PWCs to protect the feet of athletes from collisions with the soccer ball or other wheelchairs during the games. Measurement of the PWC speed during the games is an important consideration for bumper design to reduce the risk of injury and improve safety measures for the athletes [10]. Results indicate that during the soccer games most athletes maintained an average speed greater than $1 \mathrm{~m} / \mathrm{s}$. During the game, none of the participants exceeded the speed limit of power soccer, which was $2.8 \mathrm{~m} / \mathrm{s}(6.2 \mathrm{mi} / \mathrm{h})$ [7]. This speed limit was set by the Federation Internationale de Powerchair Football Association. For future research, the data logger can be vital in determining the duration of training hours and ease of maneuverability of PWCs, especially for individuals with higher levels of SCI, more involved physical disabilities, and progressive disabilities. Coaches could use speed and acceleration data to formulate training regimens based on per game requirements and the physical and functional capacities of athletes. In turn, this information may improve and refine the process of prescribing the most appropriate PWC design to power soccer athletes based on their mobility characteristics.

The limitations of this study are that all the participants were veterans and only one female consented to participate. Therefore, the results of the present study cannot be generalized to the entire PWC population participating in power soccer. Also, it might be interesting to collect speed data before the game and after the game (something we did not measure in this study), which could provide some valuable insight into how day-to-day travel in the home and community differs from PWC sporting events. Further studies are required to determine whether the distances traveled during the power soccer games differ based on the type of input control, chin-controlled versus hand-controlled joystick, or even wheelchair type. If such differences exist, then the need for classifying power soccer games on the basis of user interface may be necessary. Since 16 of the 19 study participants used PWCs with 


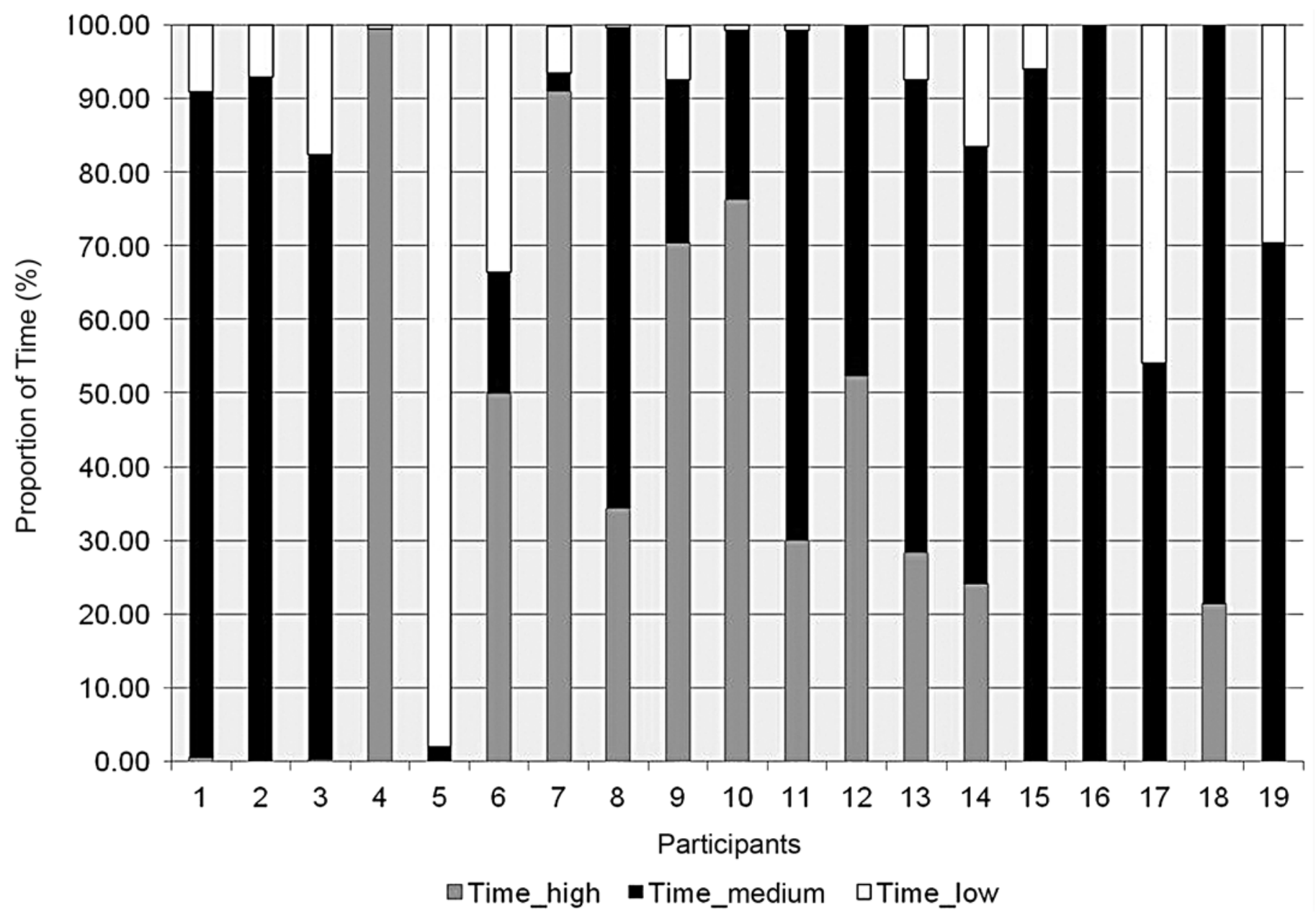

Figure 3.

Proportion of time (\%) each participant spent at three different speed levels: high (>1 m/s), medium $(0.5-1 \mathrm{~m} / \mathrm{s})$, and low $(<0.5 \mathrm{~m} / \mathrm{s})$.

rear-wheel drive, no conclusive statements can be made about the effect of PWC drive wheel on the mobility characteristics collected in this study. We did not collect data from any front-wheel drive PWCs because the data-loggerequipped caster could not be fitted to front-wheel drive PWCs. Future studies should investigate the existence of differences in mobility patterns based on drive type of PWC.

\section{CONCLUSIONS}

Despite the growth in adapted sports in the United States, little information is available regarding activity monitoring or participation of athletes with higher level impairments, specifically those using PWCs for mobility in sports and recreation. In summary, this pilot study established the efficacy of PWC data loggers to quantify the mobility characteristics of PWC use during soccer games. The results of this descriptive study may provide future insights to researchers in the field of adapted sports for people with high-level physical impairments. Also, data loggers may play a significant role in optimizing sports performance through the design of customized PWCs, programmable controls (joysticks), and types of wheelchair drive (i.e., front-wheel, mid-wheel, or rearwheel) to meet the demands of PWC games. Future studies may include electronic devices that quantify the level of increased or diminished participation in PWC sports or risks of adapted sports-related injuries such as seat shear during sports participation. 


\section{ACKNOWLEDGMENTS}

\section{Author Contributions:}

Study concept and design: R. A. Cooper, R. Cooper, M. L. Oyster. Acquisition of data: A. Kumar, A. Souza, M. L. Oyster, R. Cooper, R. A. Cooper.

Analysis and interpretation of data: A. Kumar, A. M. Karmarkar, D. M. Collins, R. A. Cooper.

Drafting of manuscript: A. Kumar, A. M. Karmarkar, A. Souza. Critical revision of manuscript for important intellectual content: M. L. Oyster, R. Cooper, R. A. Cooper, D. M. Collins.

Statistical analysis: A. Kumar, D. M. Collins, A. M. Karmarkar. Obtained funding: R. A. Cooper.

Administrative, technical, or material support: M. L. Oyster, A. Souza.

Study supervision: R. A. Cooper, R. Cooper, M. L. Oyster.

Financial Disclosures: The authors have declared that no competing interests exist.

Funding/Support: This material was based on work supported by the VA Rehabilitation Research and Development Service, Office of Research and Development (grant B3142C) and the National Science Foundation (cooperative agreement EEC-0540865).

Institutional Review: The study was approved by the VA Pittsburgh Healthcare System Institutional Review Board, and all the study participants went through the informed consent process before participating. Participant Follow-Up: The authors plan to inform participants of the publication of this study. They will include notification in the HERL Newsletter, which goes out to all of research participants who have expressed an interest in receiving it.

Disclaimer: Any opinions, findings, and conclusions or recommendations expressed in this material are those of the authors and do not necessarily reflect the views of the National Science Foundation. The contents of this article do not represent the views of the VA or the U.S. Government.

\section{REFERENCES}

1. Pasquina PF. National Disabled Veterans Winter Sports Clinic. J Rehabil Res Dev. 2006;43(7):xi-xvi. [PMID: 17436166] http://dx.doi.org/10.1682/JRRD.2006.08.0099

2. Sporner ML, Fitzgerald SG, Dicianno BE, Collins D, Teodorski E, Pasquina. PF, Cooper RA. Psychosocial impact of participation in the National Veterans Wheelchair Games and and Winter Sports Clinic. Disabil Rehabil. 2009;31(5):410-18. [PMID: 18608391]

http://dx.doi.org/10.1080/09638280802030923

3. Davis GM. Exercise capacity of individuals with paraplegia. Med Sci Sports Exerc. 1993;25(4):423-32. [PMID: 8479296]

4. Healthy people 2010. 2nd ed. Washington (DC): U.S. Department of Health and Human Services; 2010. p. 3-6.

5. Barfield JP, Malone LA, Collins JM, Ruble SB. Disability type influences heart rate response during power wheelchair sport. Med Sci Sports Exerc. 2005;37(5):718-23.
[PMID: 15870623]

http://dx.doi.org/10.1249/01.MSS.0000161807.77552.8B

6. Wu SK, Williams T. Factors influencing sport participation among athletes with spinal cord injury. Med Sci Sports Exerc. 2001;33(2):177-82. [PMID: 11224802] http://dx.doi.org/10.1097/00005768-200102000-00001

7. Federation Internationale de Powerchair Football Association [Internet]. Paris (France): FIPFA [cited 2009 Oct 25]. Available from: http://www.fipfa.org/.

8. United States Power Soccer Association [Internet]. [cited 2009 Oct 25]. Available from: http://www.powersoccerusa.net/.

9. Cooper RA, Thorman T, Cooper R, Dvorznak MJ, Fitzgerald SG, Ammer W, Song-Feng G, Boninger ML. Driving characteristics of electric-powered wheelchair users: How far, fast, and often do people drive? Arch Phys Med Rehabil. 2002;83(2):250-55. [PMID: 11833031] http://dx.doi.org/10.1053/apmr.2002.28020

10. Sibille J, Cowin LW, O’Riain MD. Wheelchair bumper in sports for the disabled. Proceedings of the 27th Annual RESNA Conference; 2004 Jun 20-22; Orlando, FL.

11. Reid G, Prupas A. A documentary analysis of research priorities in disability sport. Adapt Phys Activ Q. 1998;15(2): $168-78$.

12. Gailey RS, Cooper RA. Sports medicine for the disabled. The time for specialization in prosthetics and orthotics is now. Prosthet Orthot Int. 2009;33(3):187-19.

[PMID: 19658008]

http://dx.doi.org/10.1080/03093640903083934

13. Nolan L. Carbon fibre prostheses and running in amputees: A review. Foot Ankle Surg. 2008;14(3):125-29.

[PMID: 19083629] http://dx.doi.org/10.1016/j.fas.2008.05.007

14. Sonenblum SE, Sprigle S, Harris FH, Maurer CL. Characterization of power wheelchair use in the home and community. Arch Phys Med Rehabil. 2008;89(3):486-91. [PMID: 18295627] http://dx.doi.org/10.1016/j.apmr.2007.09.029

15. Cooper RA, Tolerico M, Kaminski BA, Spaeth D, Ding D, Cooper R. Quantifying wheelchair activity of children: A pilot study. Arch Phys Med Rehabil. 2008;87(12):977-83. [PMID: 19033759] http://dx.doi.org/10.1097/PHM.0b013e31818dfe74

16. Ferretti EC. Assessing the influence of wheelchair on individuals with spinal cord injury using a measure of participation [dissertation]. [Pittsburgh (PA)]: University of Pittsburgh; 2007. 174 p.

17. Sporner ML, Grindle GG, Kelleher A, Teodorski EE, Cooper R, Cooper RA. Quantification of activity during wheelchair basketball and rugby at the National Veterans Wehelchair Games: A pilot study. Prosthet Orthot Int. 
JRRD, Volume 49, Number 1, 2012

2009;33(3):210-17. [PMID: 19658011]

http://dx.doi.org/10.1080/03093640903051816

18. United States Paralympics Committee. Research \& Sports Science Consortium [Internet]. Colorado Springs (CO): United States Paralympics Committee; 2010 [cited 2011 Mar 5]. Available from:

http://usparalympics.org/resources/research-sports-science-consortium

Submitted for publication September 29, 2010. Accepted in revised form April 18, 2011.
This article and any supplementary material should be cited as follows:

Kumar A, Karmarkar AM, Collins DM, Souza A, Oyster ML, Cooper R, Cooper RA. Pilot study for quantifying driving characteristics during power wheelchair soccer. J Rehabil Res Dev. 2012;49(1):75-82.

http://dx.doi.org/10.1682/JRRD.2010.09.0191

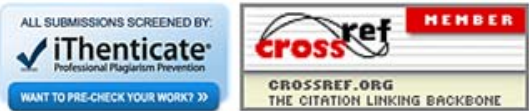

